\title{
The impact of the COVID-19 pandemic on the Greek population: Suicidal ideation during the first and second lockdown
}

\author{
ARTICLE HISTORY: Received 24 October 2021/Published Online 26 November 2021
}

More than a year has passed since World Health Organization (WHO) declared COVID-19 as a pandemic, and during this period over 237 million cases and more than 4.8 million deaths have occurred worldwide due to COVID-19. ${ }^{1}$ This unprecedented pandemic not only has burdened health systems but it also constitutes a major stressful event both due to the threat of illness and death that it poses, and to the drastic impact on human relations, financial activity, access to health services, etc. Additional factors that may contribute to stress include the protection measures against COVID-19, social distancing, and mobility restrictions.

The impact of the pandemic on suicidal behavior, especially on the Greek population, is of critical importance, due to the increase in suicidality during the recent financial crisis in the country. ${ }^{2}$ The impressive decline in the GDP during the first months of the COVID-19 pandemic (-9\% of GDP), ${ }^{3}$ unemployment, isolation, reduced social contacts, problems in accessing mental health services, and also the limitations in terms of psychological support may increase the risk of suicidal behavior. ${ }^{4}$

With a view to investigating the psychosocial effects of the COVID-19 pandemic, an online questionnaire was developed in March 2020 by the Second Department of Psychiatry of the National and Kapodistrian University of Athens (NKUA) and the Postgraduate Program "Liaison Psychiatry: Integrated Care of Physical and Mental Health" of NKUA. This questionnaire included items regarding demographic characteristics, physical and mental health data, and issues related to the pandemic and the imposed restriction measures, such as perceived changes in participants' biorhythms, habits, and relationships with their colleagues, friends, and family. In addition, participants were asked to complete psychometric scales with regard to anxiety, depression and suicidal ideation, family functioning, anger and resilience. During the first national lockdown in Greece (April 7 to May 3 ) a total of 5,748 adults from the community participated in the survey by anonymously completing the aforementioned questionnaire on a secure website of NKUA. A considerable effort was devoted to make the sample as representative as possible and to include members of the community who do not usually participate in such surveys, as individuals of older age or individuals with health conditions.

The 5.20\% two-week prevalence of suicidal ideation found in our study is an intermediate rate with respect to the $2.4 \%$ onemonth prevalence in 2008, the $6.7 \%$ in 2011 and the $2.6 \%$ prevalence in $2013 .{ }^{5}$ Among the respondents, $14.1 \%$ were potential cases of anxiety, while $26.5 \%$ of depression. Independent risk factors for suicidal ideation included anxiety, depression, impaired family functioning, being unmarried or divorced, having a mental health history, as well as a poor perceived quality of physical health. In contrast, higher resilience, positive feelings with regard to the lockdown measures, relationship with friends, and faith in a Supreme Being emerged as protective factors for suicidal ideation. ${ }^{6}$ Investigating the risk and protective factors for suicidal ideation is especially important during this difficult period of the pandemic.

There was an additional significant finding in this study: individuals who completed the questionnaire during the last two weeks of the first lockdown reported statistically significantly higher suicidal ideation, depression, and anxiety than those who completed it in the previous two weeks, while a similar finding was revealed in a study from USA. ${ }^{7}$

Therefore, we were looking forward to the results of our survey conducted during the second lockdown. ${ }^{8}$ From the 5,116 individuals who had fully completed our questionnaire with respect to suicidal ideation during the first lockdown, 811 fully completed it for the second time from November 22 to December 21, 2020. Suicidal ideation was not found significantly different compared to the first lockdown. Independent risk factors for suicidal ideation during the second lockdown were depression, anxiety, living with a person with frail health and vulnerable for COVID-19 and suicidal ideation during the first lockdown.

It is noted that during the second lockdown the rates of potential depression cases remained unchanged, whereas anxiety rates increased. Greater accessibility to health services, state financial support and increased mobility might have contributed to the stability of suicidal ideation despite the greater severity of the second wave of the COVID-19 pandemic. 
The aforementioned studies determined the prevalence of suicidal ideation and its association with various demographic, clinical, social, familial, and psychopathological factors in a cohort context at different stages of the COVID-19 pandemic, with the relevant literature being rather poor. We consider that the provision of such data is critical for the plans of health system in pandemic conditions, while this longitudinal study is in progress during the subsequent waves of the pandemic.

\author{
Rossetos Gournellis \\ Professor of Psychiatry, 2nd Department of Psychiatry, School of Medicine, \\ National and Kapodistrian University of Athens, \\ University General Hospital "Attikon" UMHRI), Athens, Greece
}

\author{
Vasiliki Efstathiou \\ Psychologist, Postgraduate Program "Liaison Psychiatry Intergrated Care of Physical \\ and Mental Health", School of Medicine, National and Kapodistrian University of Athens, Greece
}

\section{References}

1. World Health Organization. Coronavirus disease 2019 (COVID-2019): weekly epidemiological update 2021 (Retrieved: 16 October 2021). Available from: www.who.int/emergencies/diseases/novel-coronavirus2019/situation-reports

2. Economou M, Angelopoulos E, Peppou LE, Souliotis K, Stefanis C. Suicidal ideation and suicide attempts in Greece during the economic crisis: an update. World Psychiatry 2016, 15:83-84, doi: 10.1002/wps.20296

3. Hellenic Statistical Authority (ELSTAT). 2nd estimate of the Annual National Accounts for the year 2020 (provisional data) 2021 (Retrieved: 16 October 2021). Available from: https://www.statistics.gr/documents/ 20181/77f9e97d-79ac-6cb5-a378-fd45be939923

4. Reger MA, Stanley IH, Joiner TE. Suicide mortality and coronavirus disease 2019-a perfect storm? JAMA Psychiatry 2020, 77:1093-1094, doi: 10.1001/jamapsychiatry.2020.1060
5. Fountoulakis KN. Suicides in Greece before and during the period of austerity by sex and age group: relationship to unemployment and economic variables. J Affect Disord 2020, 260:174-182, doi: 10.1016/j.jad. 2019.09.001

6. Papadopoulou A, Efstathiou V, Yotsidi V, Pomini V, Michopoulos I, Markopoulou E et al. Suicidal ideation during COVID-19 lockdown in Greece: prevalence in the community, risk and protective factors. Psychiatry Res 2021, 297:113713, doi: 10.1016/j.psychres.2021.113713

7. Killgore WDS, Cloonan SA, Taylor EC, Allbright MC, Dailey NS. Trends in suicidal ideation over the first three months of COVID-19 lockdowns. Psychiatry Res 2020, 293: 113390, doi: 10.1016/j.psychres.2020. 113390

8. Efstathiou V, Michopoulos I, Yotsidi V, Smyrnis N, Zompola C, Papadopoulou $A$ et al. Does suicidal ideation increase during the second COVID-19 lockdown? Psychiatry Res 2021, 301:113990, doi: 10.1016/j. psychres.2021.113990 